\title{
The scattering of $\mathrm{SH}$ waves by a finite crack with a superposition based diffraction technique
}

\author{
Camilo Valencia \\ Advisor: \\ Juan David Gomez \\ Departamento de Ingeniería Civil \\ Universidad EAFIT \\ Medellín, \\ Colombia
}

June 10, 2014

\begin{abstract}
The problem of diffraction of cylindrical and plane $S H$ waves by a finite crack is revisited. We construct an approximate solution by the addition of independent diffracted terms. We start with the derivation of the fundamental case of a semi-infinite crack obtained as a degenerate case of generalized wedge. This building block is then used to compute the diffraction of the main incident waves. The interaction between the opposite edges of the crack is then considered one term at a time until a desired tolerance is reached. We propose a recipe to determine the number of required interactions as a function of frequency. The solution derived with the superposition technique can be applied at low and high frequencies.

Keywords: wave propagation, wave scattering, frequency domain analysis, diffraction, SH waves.
\end{abstract}

\section{Introduction}

The determination of the scattered field produced by an elastic wave incident upon a crack of finite length is of fundamental relevance in many different problems of physics and engineering. For instance, cracks are frequently encountered at the interior of homogeneous media in the form of defects in machine parts (Garnier et al., 2011), as faults at the interior of the earth crust (Murai, 2007) or as cavities enclosing fossil energy reservoirs. In all of these cases, efficient engineering applications or understanding of physical phenomena, are strongly dependent on the level of accuracy and on the correct interpretation of results from the wave scattering problem associated to the crack problem. Although only in rare cases the existing cracks appear isolated, knowing and understanding the solution to the diffraction caused by 
a single crack plays a key role in these most general scenarios. This paper revisits the well known problem of scattering and diffraction of horizontally polarized $S H$ waves caused by a crack of finite length at the interior of an isotropic elastic full-space. Despite the fact that several studies have been conducted in the subject, alternative solutions are useful as they bring further understanding of the problem. In this work we find an approximate solution to the field scattered by a finite crack upon incident plane and cylindrical $S H$ waves. The solution is found using superposition of fields contributed by different diffraction sources of the Sommerfeld's type and although it takes the form of an infinite series, in our approach accurate approximations can be obtained retaining just a few terms in the series without dealing with convergecy analysis. Moreover, the solution is built following a physical reasoning which is attractive for the analyst.

The problem of scattering of plane $S H$ waves by cracks has been widely studied from a numerical and an analytical point of view. A review of seminal works in the subject can be found in Mow \& Pao (1971) and in Achenbach et al. (1982). A landmark contribution of analytic nature is identified in the work by Sommerfeld (1896) who derived the solution for the diffraction of $S H$ waves induced by a semi-infinite crack. That work was also relevant since it opened the door to a number of studies dealing with more general cases. For instance SánchezSesma \& Iturrarán-Viveros (2001), performing superposition of Sommerfeld's solution, found an analytic expression for the diffracted field produced by of a plane $S H$ wave incident upon a single crack of finite size. In that solution the first diffracted waves generated at the tips were limited to propagate as plane waves when interacting with the opposite tip of the crack, thus neglecting its original cylindrical nature. However, these authors showed that the resulting inaccuracies were only noticeable at low frequencies where the crack opening displacement (COD) was small. This solution was later extended in Iturrarán-Viveros et al. (2010) to consider also the case of an incident cylindrical wave and both solutions have since then been used by various authors to validate numerical methods, (Krüger et al., 2005).

An analytical solution in the time domain was also found by De Hoop (2000), who derived a closed-form expression for the scattering of a plane $S H$ wave introduced by a generalized linear slip fracture of finite length in the Kirchhoff approximation. Similarly, Caleap et al. (2007) found an analytic expression for the angular function proposed by Waterman \& Truell (1961) in order to describe the far field scattered by a single crack. In more recent contributions the problem was also solved by Tsaur (2010) using a region matching technique leading to the treatment in terms of separation of variables and by Chen et al. (2013) who solved it using a set of boundary integral equations, while recent numerical approaches based upon direct (and indirect) boundary element methods and finite difference schemes are identified in Iturrarán-Viveros et al. (2005); Pérez-Ruiz et al. (2007); Chen et al. (2012).

On the other hand, the interpretation of a semi-infinite crack as a particular instance of a generalized semi-infinite wedge, created the possibility of adapting solutions previously derived for wedges within the context of electromagnetic waves to the scattering of elastic fields. For instance MacDonald (1902) obtained a series expansion for the total field in a wedge under plane and cylindrical incident waves in terms of Bessel functions. This solution was later re-derived by Sanchez-Sesma (1990) and used in Sánchez-Sesma \& Iturrarán-Viveros (2001) as a measurement of the level of error introduced by the plane wave approximation introduced in the secondary diffracted waves. Similarly, Hudson (1963) studied the wedge under a line source of harmonic $S H$ waves for combined Dirichlet and Neumann boundary conditions and expressed his solution in the form of an infinite integral, while Abo-Zena \& King (1973) solved the problem of a wedge under a line source applied along its free surface. In that solution the response was separated in terms of the incident, reflected and diffracted fields, but the total diffracted term was given as a singular integral. The idea of separating the total field in a wedge into terms related to the incident, reflected and diffracted fields 
had already been used in the geometrical theory of diffraction (GTD) from Keller (1957, 1956, 1962). That approach was later improved by Kouyoumjian \& Pathak (1974) who found the diffracted field for a generalized wedge under incident plane and cylindrical fronts in terms of diffraction coefficients similar to those used in the solution of reflection-transmission problems.

The superposition technique, based on the addition of incident, reflected and diffracted waves was recently generalized by Jaramillo et al. (2013), where it was used in the solution of scattering of plane $S H$ waves incident against canyons of arbitrary shape. Because the method is based on the addition of diffraction terms, it has been given the name superposition based diffraction (SBD). Its main distinguishing feature is the fact that it results in a series solution, where high levels of accuracy can be reached even when only a few terms are retained. In other words, in the superposition based diffraction technique there is a direct relation among the infinite terms in the series and actual diffraction events and the number of required terms can be shown to be frequency dependent. In this work we use this superposition approach and construct the solution like in Sánchez-Sesma \& Iturrarán-Viveros (2001), where the finite crack is obtained as the superposition of two semi-infinite cracks. While these authors used as starting point the fundamental solution by Sommerfeld (1896), accounting only by the diffraction of plane waves, we started from the generalized wedge solved by Kouyoumjian \& Pathak (1974) which directly considers the initially diffracted waves as cylindrical fronts. Our solution is also different since we find the displacement field everywhere, and not only the crack opening displacement. The physical character contained in the solution process makes the solution very appealing for the validation of numerical implementations.

In the article we first describe the canonical solution by Kouyoumjian \& Pathak (1974), corresponding to the diffraction of electromagnetic waves by a generalized wedge. That solution is used here to derive the contribution from the diffracted field which is used to build the solution to the diffraction by a finite crack after its recursive application in terms of primary and higher order diffraction terms. The obtained solution, valid for cylindrical and plane waves is then simplified and presented in terms of a simple expression valid for the particular case of normal incidence. Since the proposed solution takes the form of an infinite series, we propose a recipe to determine the number of required terms, given the frequency and a predefined tolerance. It is found that accurate solutions are found with just a few terms in the series. In order to validate our solution to the crack problem, we compare our frequency and time domain results with those obtained via a numerical boundary element method.

\section{Diffraction by a semi-infinite crack as a particular instance of the diffraction by a generalized wedge.}

In this section we describe the canonical problem forming the basis of the superposition based diffraction technique (SBD). The problem domain and incident wave field is defined

in fig. 1. It consists of a generalized wedge with traction's free surfaces intercepting at a corner singularity of external angle $\nu \pi$ where $\nu$ is a factor that completely defines the wedge varying between 0.0 and 2.0. The generalized wedge is submitted to an incident plane front characterized by its propagation direction and forming an angle $\phi^{\prime}$ with the free surface of the wedge. In fig. 1, regions of existence (or absence) of incident and reflected rays are fully described and denoted by the common jargon of geometrical theory of diffraction like illuminated (or shadow) zones.

The lines separating the different regions of existence of incident and reflected rays, and denoted in the figure like reflection and incidence boundaries, represent regions of discontinuity 


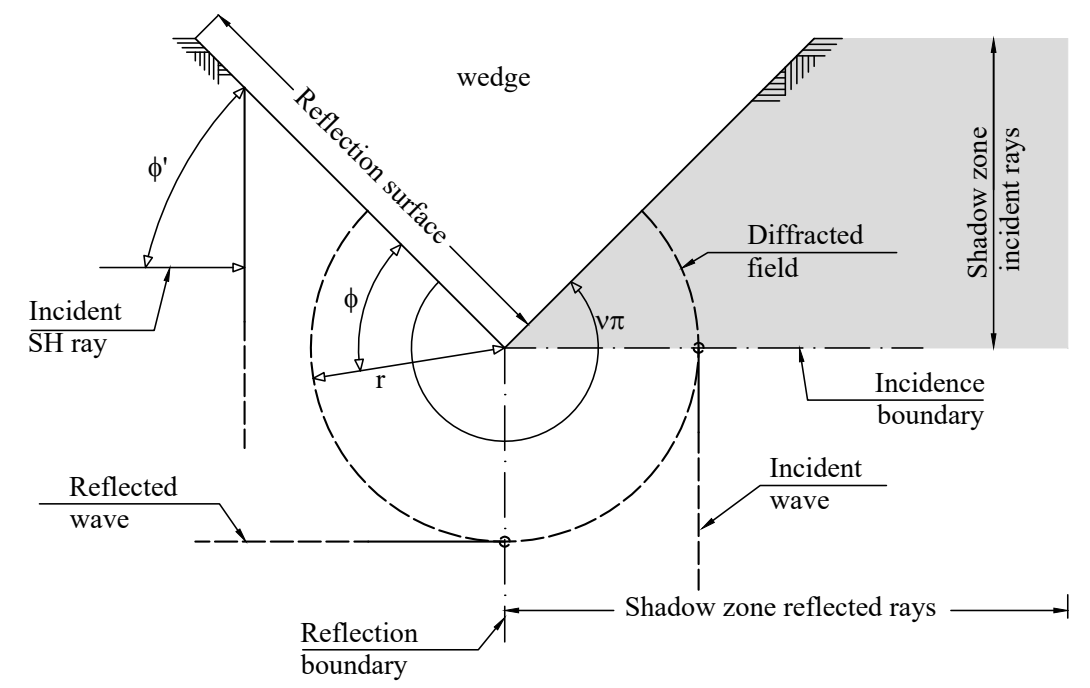

Figure 1: Plane wave incident against a generalized wedge

of each term considered independently. The diffraction field generated by the corner singularity corresponds to a cylindrical wave that penetrates into the shadow zone and restores continuity along these reflection and incidence boundaries. In summary, the construction process to find the solution for a single wedge amounts to finding the total field contributed by the incident and reflected fields $w^{i n c}$ and $w^{r}$, which are discontinuous or even absent from certain regions, plus the consideration of the diffracted field $w^{D}$ generated at the corner singularity and restoring continuity everywhere, i.e., the term $w^{D}$ provides the proper transition between the illuminated and the shadow zones.

In the proposed methodology, the contribution of the diffracted field is taken directly from the approximate solution proposed by Kouyoumjian \& Pathak (1974) and corresponding to a generalized infinite wedge submitted to an incident plane or cylindrical wave. The case of a cylindrical diffraction front may correspond to a previously diffracted field or to a primary incident cylindrical wave. The generalized solution for the diffracted field is given in eq. (1) for a field point with coordinates $(\phi, r)$ as follows

$$
\begin{aligned}
w^{D}(\phi, r) & =A \frac{-e^{(-\hat{i}(k r+\pi / 4))}}{2 \nu \sqrt{2 \pi} \sqrt{k r}}\left[\cot \left(\frac{\pi+\left(\phi-\phi^{\prime}\right)}{2 \nu}\right) F\left(k L b^{+}\left(\phi-\phi^{\prime}\right)\right)\right. \\
& +\cot \left(\frac{\pi-\left(\phi-\phi^{\prime}\right)}{2 \nu}\right) F\left(k L b^{-}\left(\phi-\phi^{\prime}\right)\right) \\
& +\cot \left(\frac{\pi+\left(\phi+\phi^{\prime}\right)}{2 \nu}\right) F\left(k L b^{+}\left(\phi+\phi^{\prime}\right)\right) \\
& \left.+\cot \left(\frac{\pi-\left(\phi+\phi^{\prime}\right)}{2 \nu}\right) F\left(k L b^{-}\left(\phi+\phi^{\prime}\right)\right)\right]
\end{aligned}
$$

where $r=$ radial coordinate to the field point measured from the vertex of the wedge, $\phi=$ angular coordinate measured with respect to the reflection boundary, $\phi^{\prime}=$ incidence angle measured with respect to the reflection boundary, $\nu \pi=$ wedge angle with $0.0 \leq \nu \leq 2.0$, $r^{\prime}=$ radius of the incident cylindrical wave (for the diffraction of a cylindrical front), $k=$ wave number and $\beta=$ velocity of wave propagation. The remaining terms appearing in eq. (1) are 
defined as

$$
\begin{aligned}
& F(X)=2 \hat{i} \sqrt{X} e^{\hat{i} X} \int_{\sqrt{X}}^{\infty} e^{-\hat{i} \tau^{2}} \mathrm{~d} \tau \\
& L=r \quad \text { for incident plane waves } \\
& L=\frac{r r^{\prime}}{r+r^{\prime}} \quad \text { for incident cylindrical waves } \\
& b^{ \pm}(\theta)=2 \cos ^{2}\left(\frac{2 \nu \pi Z^{ \pm}-\theta}{2}\right) \\
& Z^{+}=\left\{\begin{array}{ll}
0 & \text { if } \theta \leq \nu \pi-\pi \\
1 & \text { if } \theta>\nu \pi-\pi
\end{array}, \quad Z^{-}= \begin{cases}-1 & \text { if } \theta<\pi-\nu \pi \\
0 & \text { if } \pi-\nu \pi \leq \theta \leq \pi+\nu \pi \\
1 & \text { if } \theta>\pi+\nu \pi\end{cases} \right.
\end{aligned}
$$

From the solution described in eq. (1) it is clear that the diffraction yields a cylindrical front and that for values of $\phi$ corresponding to points far removed from the incidence and reflection boundaries, the amplitude decays with $1 / \sqrt{k r}$. On the other hand, right at the incidence/reflection boundary the diffracted field is discontinuous. This is a required condition that must be satisfied by the term $w^{D}$ in order to match the corresponding discontinuity appearing in the superposition of the incident and reflected contributions.

\section{Diffraction by a finite crack via superposition of diffraction sources.}

In order to obtain the solution to the diffraction of incident $S H$ waves by a single crack we start from the solution to the problem shown in fig. 1 and given in eq. (1). If the angular factor is given the particular value of $\nu=2.0$, the generalized wedge collapses into a zero-thickness semi-infinite crack with traction free surfaces. Two such cracks, defined in $y \in(-\infty, a / 2]$ and $y \in[-a / 2, \infty)$ can then be superimposed to yield a single crack of length $l=a$ defined by the domains of intersection of the two fundamental cracks; i.e., in $y \in[-a / 2, a / 2]$. The above superposition scheme is described in fig. 2 where we also show the specific parameters that must be applied in eq. (1) in order to consider incidence of plane and cylindrical fronts. 


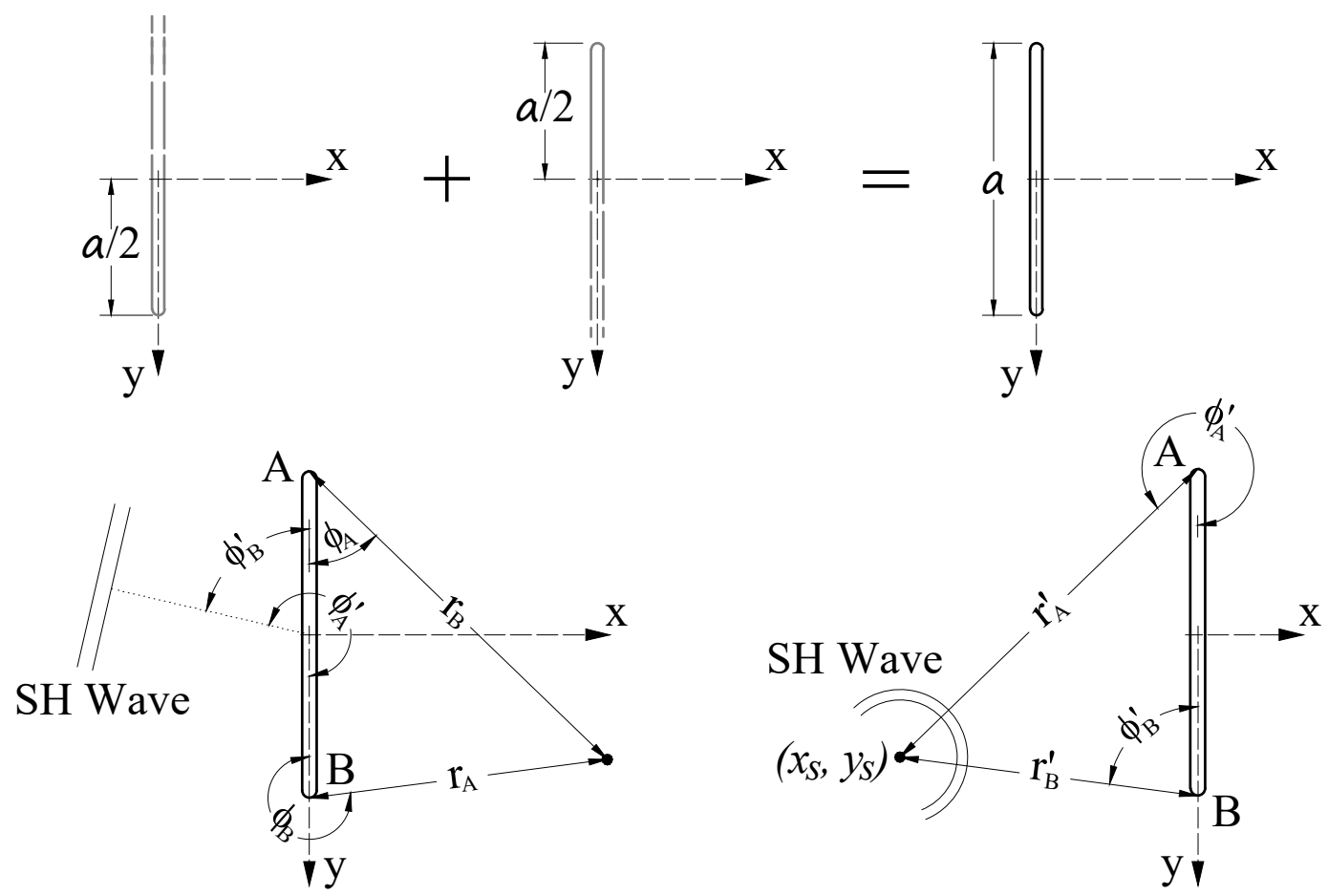

Figure 2: Problem definition and construction of the solution for a finite crack via superposition of fundamental semi-infinite cracks.

The incident wave is defined by

$$
w^{i n c}=w_{0} e^{\left[i k x \sin \left(\phi_{B}^{\prime}\right)+i k y \cos \left(\phi_{B}^{\prime}\right)\right]}
$$

for plane incident wave, and

$$
w^{i n c}=\frac{w_{0}}{\sqrt{R}} e^{(i k R)}
$$

for cylindrical incident wave, with $R$ measured from the source point $\left(x_{s}, y_{s}\right)$ and with $w_{0}$ being the amplitude at $R=1.0$.

Using a plane or cylindrical incident wave and denoting the reflected field by $w^{r}$, the solution in the illuminated zone takes the form;

$$
w^{T}=w^{i n c}+w^{r}+w_{A}+w_{B}+w_{A-B}+w_{B-A}+w_{A-B-A}+w_{B-A-B}+\ldots
$$

while in the shadow zone of the incident and reflected rays it reads;

$$
w^{T}=w_{A}+w_{B}+w_{A-B}+w_{B-A}+w_{A-B-A}+w_{B-A-B}+\ldots
$$

and

$$
w^{T}=w^{i n c}+w_{A}+w_{B}+w_{A-B}+w_{B-A}+w_{A-B-A}+w_{B-A-B}+\ldots
$$

respectively.

In the expressions above the extreme subscripts refer to the first and last source of diffraction. For instance, the term $w_{B-A-B-A}$ indicates a diffracted wave that originated at point $B$ and subsequently experienced diffraction at $A, B$ and $A$ again. 
In order to obtain the total diffracted field it is necessary to apply eq. (1) recursively. After some mathematical manipulations the final solution for the diffracted field can be written like;

$$
w^{D}\left(\phi_{A}, \phi_{B}, r_{A}, r_{B}\right)=A_{A} \sum_{n=1}^{N} V_{A} \mathbf{G}\left(\phi_{A}, \phi_{A}^{\prime}, r_{A}, L_{A}\right)+A_{B} \sum_{n=1}^{N} V_{B} \mathbf{G}\left(\phi_{B}, \phi_{B}^{\prime}, r_{B}, L_{B}\right)
$$

with

$$
V_{\bullet}= \begin{cases}1 & \text { if } n=1 \\ \mathbf{G}(0.0,0.0, n a, L \bullet) & \text { if } n>1\end{cases}
$$

where

$$
\begin{aligned}
\mathbf{G}\left(\phi, \phi^{\prime}, r, L\right) & =\frac{-e^{(-\hat{i}(k r+\pi / 4))}}{4 \sqrt{2 \pi} \sqrt{k r}}\left[\cot \left(\frac{\pi+\left(\phi-\phi^{\prime}\right)}{4}\right) F\left(k L b^{+}\left(\phi-\phi^{\prime}\right)\right)\right. \\
& +\cot \left(\frac{\pi-\left(\phi-\phi^{\prime}\right)}{4}\right) F\left(k L b^{-}\left(\phi-\phi^{\prime}\right)\right) \\
& +\cot \left(\frac{\pi+\left(\phi+\phi^{\prime}\right)}{4}\right) F\left(k L b^{+}\left(\phi+\phi^{\prime}\right)\right) \\
& \left.+\cot \left(\frac{\pi-\left(\phi+\phi^{\prime}\right)}{4}\right) F\left(k L b^{-}\left(\phi+\phi^{\prime}\right)\right)\right]
\end{aligned}
$$

and

$$
L_{\bullet}= \begin{cases}r_{\bullet} & \text { if } n=1, \text { and plane incidence } \\ r_{\bullet} r_{\bullet}^{\prime} /\left(r_{\bullet}+r_{\bullet}^{\prime}\right) & \text { if } n=1, \text { and cylindrical incidence } \\ r_{\bullet} a /\left(r_{\bullet}+a\right) & \text { other case }\end{cases}
$$

In the above, the subscript • may take the specific values $A$ or $B$ corresponding to the crack tips. Accordingly in eq. (5) $\phi_{\bullet}$ and $r_{\bullet}$ represent the angular and radial coordinates of a field point measured with respect to the crack tips $A$ and $B, r_{\bullet}^{\prime}$ is the radius of the incident cylindrical wave also measured from each tip of the crack. $A_{\bullet}$ is the amplitude and phase of the incident front upon arrival to a given crack tip, the parameter $N$ indicates the number of terms considered in the series. The contribution from the incident fronts at the tips are given by;

$$
\begin{gathered}
\text { Plane incident wave }\left\{\begin{array}{l}
A_{A}=w^{i n c} e^{\left[i k a \cos \left(\phi_{B}^{\prime}\right) / 2\right]} \\
A_{B}=w^{i n c} e^{\left[-i k a \cos \left(\phi_{B}^{\prime}\right) / 2\right]}
\end{array}\right. \\
\text { Cylindrical incident wave }\left\{\begin{array}{l}
A_{A}=w^{i n c} e^{\left(-i k r_{A}^{\prime}\right)} \\
A_{B}=w^{i n c} e^{\left(-i k r_{B}^{\prime}\right)}
\end{array}\right.
\end{gathered}
$$

Accordingly, the exact solution corresponds to the case $N \rightarrow \infty$. This parameter has the physical meaning of number of diffraction sources considered in the series. Every increment of this parameter implies the consideration in the series of a new diffracted wave, travelling from one crack tip to the other. Each additional considered wave has an amplitude which is smaller than its parent wave. This relation between the number of terms appearing in the series and the number of diffraction sources allows the solution to be extended to any desired accuracy depending on the value of the dimensionless frequency parameter $\eta=\frac{a}{\lambda}$ relating the crack length to the incident wave length. 


\section{Particular solution for plane incidence at $\phi_{B}^{\prime}=\pi / 2$.}

In the particular case of normal incidence (i.e., $\phi_{B}^{\prime}=\pi / 2$ ) and due to the geometric symmetry of the problem, terms like $A_{A}$ and $A_{B}$ in eq. (5) take equivalent values. Thus the diffracted field simplifies into:

$w^{D}\left(\phi_{A}, \phi_{B}, r_{A}, r_{B}\right)=A_{A} \sum_{n=1}^{N}\left(\left(\frac{\left.-e^{(-\hat{i}(k a+\pi / 4)}\right)}{2 \sqrt{\pi} \sqrt{k a}} F(k a)\right)^{n-1}\left[-\mathbf{G}\left(\phi_{A}, r_{A}, L_{A}\right)+\mathbf{G}\left(\phi_{B}, r_{B}, L_{B}\right)\right]\right)$

where

$$
\begin{aligned}
\mathbf{G}(\phi, r, L) & =\frac{\left.-e^{(-\hat{i}(k r+\pi / 4)}\right)}{4 \sqrt{2 \pi} \sqrt{k r}}\left[\cot \left(\frac{\pi / 2+\phi}{4}\right) F\left(k L b^{+}(\phi-\pi / 2)\right)\right. \\
& +\cot \left(\frac{3 \pi / 2-\phi}{4}\right) F\left(k L b^{-}(\phi-\pi / 2)\right) \\
& +\cot \left(\frac{3 \pi / 2+\phi}{4}\right) F\left(k L b^{+}(\phi+\pi / 2)\right) \\
& \left.+\cot \left(\frac{\pi / 2-\phi}{4}\right) F\left(k L b^{-}(\phi+\pi / 2)\right)\right]
\end{aligned}
$$

with

$$
L_{\bullet}= \begin{cases}r_{\bullet} & \text { if } n=1 \\ r_{\bullet} a /\left(r_{\bullet}+a\right) & \text { if } n>1\end{cases}
$$

Once again the parameter $N$ limiting the series depends on the value of the dimensionless frequency $\eta$ and the pre-established level of accuracy. At larger values of $\eta$, relatively small values of $N$ are required. Physically, this means that at larger values of $\eta$ the solution corresponding to a desired accuracy is achieved with less diffraction orders.

\section{A recipe to determine the diffraction order $N$ required for a predefined tolerance.}

We now propose a procedure that can be used to estimate the number $N$ of diffraction orders that must be considered in eq. (5), given a value of the dimensionless frequency parameter $\eta$ and a predefined tolerance tol. This tolerance is defined like a percentage of the amplitude $A_{M}$ of the incident front, once it arrives to a specified crack tip. The amplitude must be selected like $A_{M}=\max \left(\left|A_{A}\right|,\left|A_{B}\right|\right)$ where $A_{A}$ and $A_{B}$ have been defined previously. The procedure guarantees that the smallest diffracted wave contained in the solution will have an amplitude smaller or equal to tol $\times A_{M}$. The procedure is listed as follows:

1. Define a value of the dimensionless frequency parameter $\eta$ and tolerance tol.

2. Plot the complex amplitude function

$$
A(r)=\frac{-e^{[-i(k r+\pi / 4)]}}{\sqrt{2 \pi} \sqrt{k r}} F(2 k r)
$$


with

$$
k=\frac{2 \pi \eta}{a} .
$$

The function $A(r)$ represents the amplitude of the transfer function of the first order diffraction produced by a plane front interacting with a tips and travelling towards the shadow zone. We have selected the amplitude function for a plane front since this wave always contains values greater than those in a cylindrical wave. Therefore, if the tolerance is reached for a plane wave, it is also valid for the analogous cylindrical wave. For cases in which $a<r<2 a$, the result corresponds to the second order diffraction. In general, for values in which $(n-1) a<r<n a$, the result for the amplitude corresponds to the $n$-th order diffraction.

3. From the previous plot, identify the value of $r$ for which $A(r) \leq t o l$.

4. Use the previously identified value of $r$ to compute $N$ like;

$$
N=\operatorname{int}(r / a)+1
$$

where $\operatorname{int}(\bullet)$ is the integer part function.

The procedure is illustrated with reference to fig. 3 where we show the variation of $A(r)$ vs $r$ for $\eta=3.0, t o l=0.05, a=1.0$ and $A_{M}=1.0$. The red line indicates the threshold under which tol has already been reached. In this case the obtained number of diffraction orders is $N=4$. This implies that if the solution is constructed with up to 4-th order diffraction terms, there will not be any diffracted waves with amplitude greater tan 0.05 .

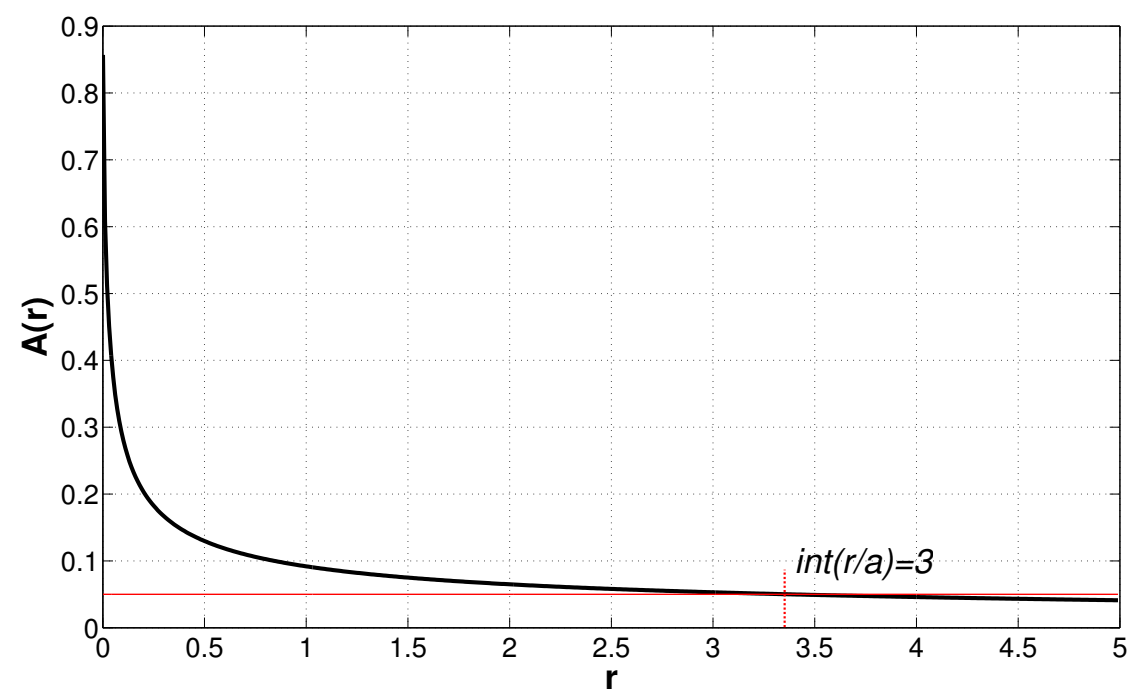

Figure 3: Variation with distance of the amplitude function $A(r)$ for a diffracted wave used to determine the maximum order of diffraction $N$ required in order to reach a specific tolerance tol given the dimensionless frequency $\eta$. 


\section{Results}

We now validate our current solution to the problem of scattering of $S H$ waves by a finite crack constructed by a diffraction based superposition approach. To that end we obtained low $(\eta=0.5)$ and high $(\eta=5.0)$ frequency results for incident plane and cylindrical waves. Our SBD-solution was compared with numerical results from a direct boundary element method (BEM). The study was conducted in the frequency and time domain. Since in the SBD-solution the only approximation is in the diffracted field, we focused on this specific component of the response by studying the transfer functions over the whole frequency range along the crack surface in the shadow zone. On the other hand, the validation in the time domain was conducted in terms of synthetic seismograms, also for receivers over the shadow zone and in terms of snapshots of the propagation patterns over the complete computational domain. As an additional validation we also compared our results with those obtained by Sánchez-Sesma \& Iturrarán-Viveros (2001), where the diffracted waves were assumed as plane fronts neglecting its cylindrical nature. The analyses were first conducted in the frequency domain, where we computed the transfer functions between the total response and the incident wave. These TFs were subsequently fast Fourier transformed in order to derive time domain results after applying a Ricker pulse defined by $R(t)=(2 \pi \tau-1) e^{-\pi \tau^{2}}$, where $\tau=f_{c}\left(t-t_{\text {ini }}\right)$ with $f_{c}=$ central frequency (or equivalently in terms of $\eta_{c}=a f_{c} / \beta$ ) and $t_{\text {ini }}=$ initial time for the intense phase. On the other hand, the results at low and high frequencies were obtained with diffraction orders of $N=5$ and $N=3$ respectively. It must be recalled that every additional term in the series implies cylindrical waves emanating from each diffraction source. In this work we considered a crack of unit length, located at $y=[-a / 2, a / 2]$ (see fig. 2) and embedded in a homogeneous elastic infinite medium with mass density $\rho=1.0$ and a shear wave propagation velocity $\beta=1.0$.

In order to show the convergency properties in our series solution, we display in fig. 4 contour maps for the amplitude of the TF for a set of receivers located over the shadow zone, where only the diffracted field is present and for a range of values of the dimensionless frequency parameter $\eta=(0,4]$. The receivers are located over the line $y=[-a / 2, a / 2]$ and the results in each case correspond to the relative error between the SBD method and the BEM algorithm computed as $|B E M-S B D| /|B E M|$. 
Cylindrical incidence

$\left(x_{s}, y_{s}\right)=(-1.0,0.0)$
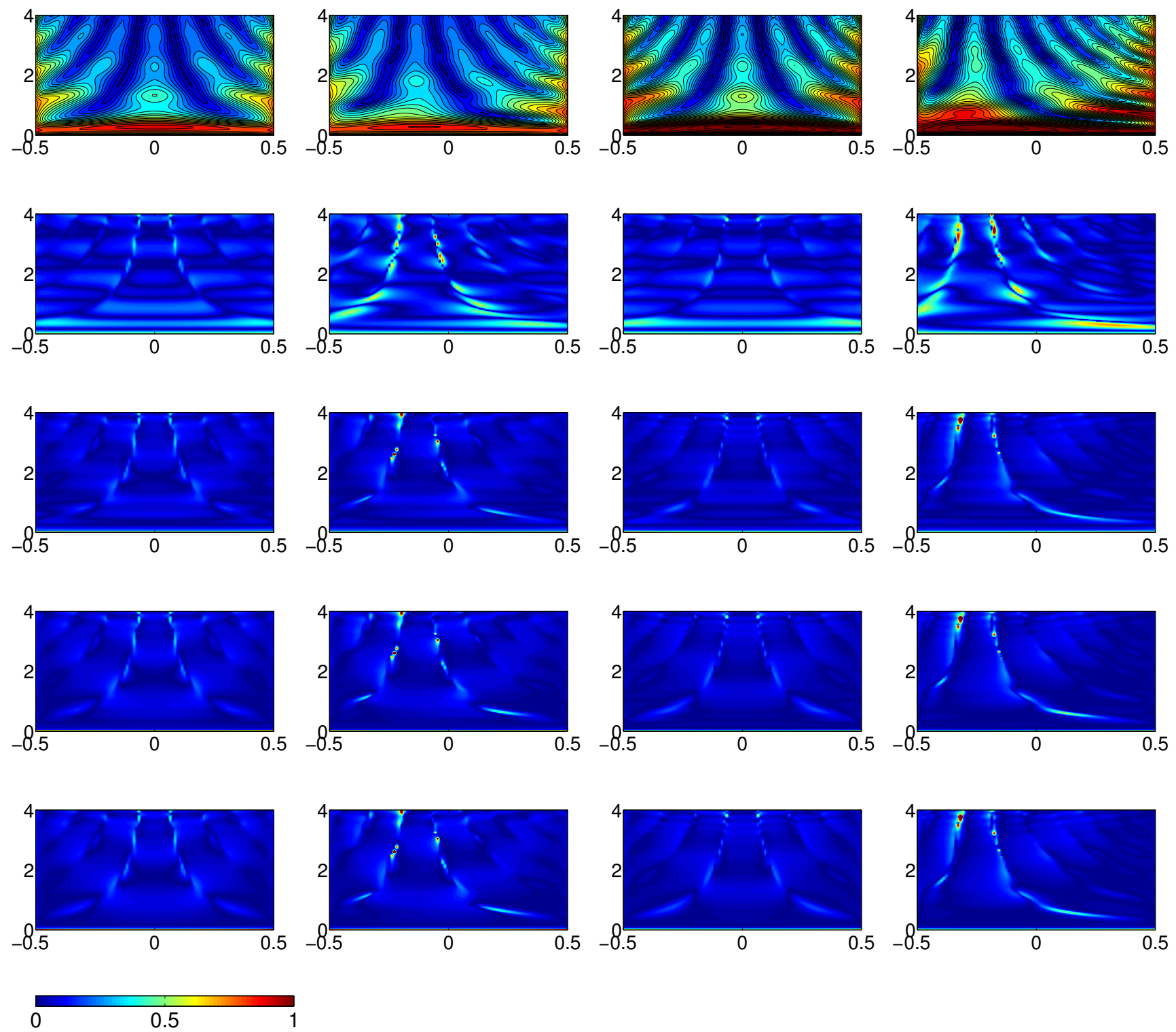

Figure 4: Frequency domain contour maps for the amplitude of the transfer function for a set of receivers over the shadowed zone of the crack surface. The SBD-contours (row 2 to 5) show the relative error between this method and the BEM solution for orders of diffraction ranging from $N=1$ to $N=4$.

The first row of contour maps in fig. 4 displays the numerical solution for the total field, while the subsequent 4 rows show the relative error between the SBD and BEM numerical results for increasing orders of diffraction ranging from $N=1$ to $N=4$. As expected, the results neglecting the interaction between the crack tips, corresponding to a single diffraction term as shown in row 2, exhibit large differences with respect to the numerical algorithm. This difference is stronger in the low frequency regime since at those frequencies the amplitude of the first diffracted wave is still very large and it requires several higher order interactions 
before their contribution can be neglected. However, it is interesting to observe how at high frequencies a good approximation is obtained by the SBD method even with a single diffraction order. This low level of interaction between the two diffraction sources at high frequencies, is evident from the $1 / \sqrt{k r}$ decay present in eq. (1). On the other hand, when 3 -rd and 4-th order diffraction is considered in the SBD solution, accuracy is maintained at high and low frequencies.

An interesting result is observed from the numerical solution for the total field associated to the responses displayed in the first and third columns for cylindrical and plane fronts under normal incidence, and for those from the second and fourth columns for cylindrical and plane fronts under an asymmetrical incidence. It is observed how, regardless of the type of incident wave the response in the shadow zone, having only the contribution from the diffracted field, is very similar in amplitude and spatial distribution for the cylindrical and plane incidence. This independence of the response in the shadow zone from the type of incident wave, is the result of the solution over this zone being contributed by the addition of cylindrical sources located at the tips.

Figure 5 displays snapshots of the propagation patterns for the case of plane waves incident with $\theta_{b}=90^{\circ}$ and $\theta_{b}=120^{\circ}$ over computational domains of size $10 \times 10$ and $3 \times 3$. The excitation in each case is a Ricker pulse with low and high characteristic frequencies and with an SBD-solution obtained with diffractions of 5-th and 3-rd order for the low and high frequency computations respectively. Each frame in the figure, qualitatively compares the results obtained with the SBD approach (left) and those from the BEM algorithm (right) at different instants of time. Although these comparisons in the time domain are highly qualitative, several observations indicating the validity of the SBD technique can be made. For instance, in every case, and as a result of the interaction between the tips of the crack, cylindrical fronts emanating from each diffraction source can be observed after the advancement of the incident front. It is also observed how the incident front is progressively recovered over the shadow zone of the incident rays. This recovery is completely achieved by the diffracted field. Moreover, this observation, regarding the recovery effect of the diffracted part of the response, is especially evident in the low frequency regime, where intuitively it should take place at shorter distances from the crack . Theoretically, at $t=\infty$ the main front should be fully recovered whereas the reflected wave should have completely vanished, also as a result of the diffracted component.

The same set of analyses were conducted for the case of incident cylindrical waves. The comparison in terms of snapshots of the propagation patterns computed with both methods are displayed in fig. 6 for the same domains used in the case of plane wave incidence. The sources for the cylindrical waves were located at the positions $\left(x_{s}, y_{s}\right)=(-2.0,0.3)$ and $\left(x_{s}, y_{s}\right)=(-1.0,0.3)$ in order to introduce asymmetric conditions in the problem. Like in the case of incident plane waves, at high frequencies it is observed how the diffracted field exhibits negligible values once it reaches the opposite tip of the crack yielding good results with only 3 orders of diffraction considered in the series. Similarly, the results also reveal the property of the diffracted field of restoring continuity of the solution over the shadow zone. 


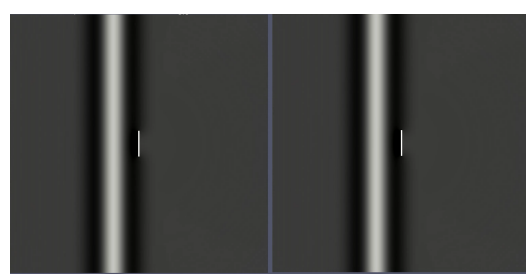

$\theta_{b}=90^{\circ}, t=5.88 \mathrm{~s}$

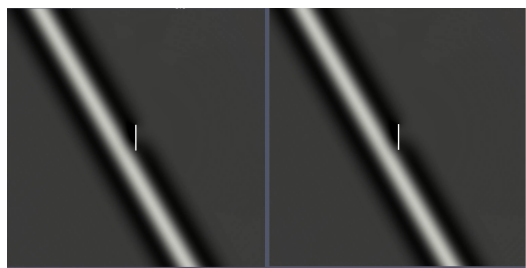

$\theta_{b}=120^{\circ}, t=5.88 \mathrm{~s}$

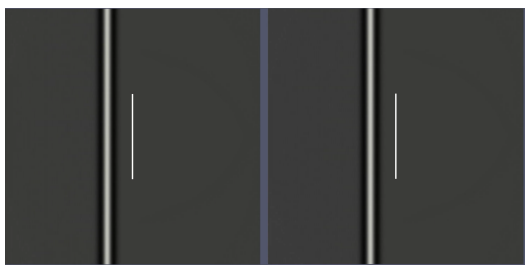

$\theta_{b}=90^{\circ}, t=1.375 \mathrm{~s}$

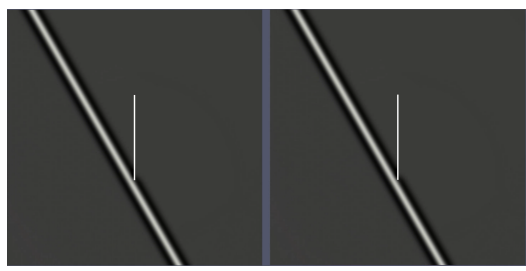

$\theta_{b}=120^{\circ}, t=1.375 \mathrm{~s}$

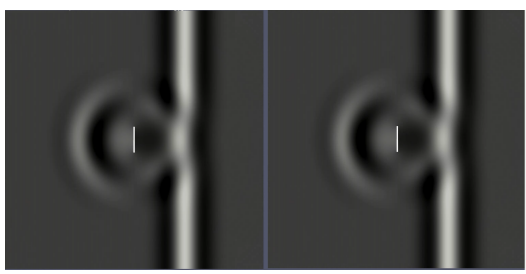

$\theta_{b}=90^{\circ}, t=8.78 \mathrm{~s}$

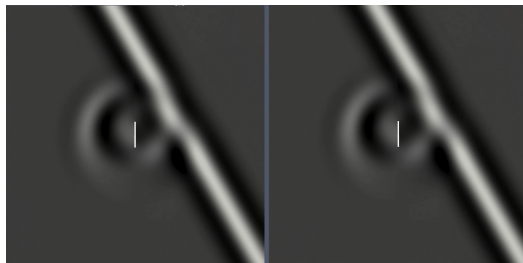

$\theta_{b}=120^{\circ}, t=8.78 \mathrm{~s}$

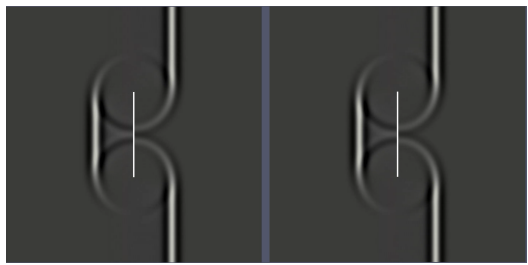

$\theta_{b}=90^{\circ}, t=2.125 \mathrm{~s}$

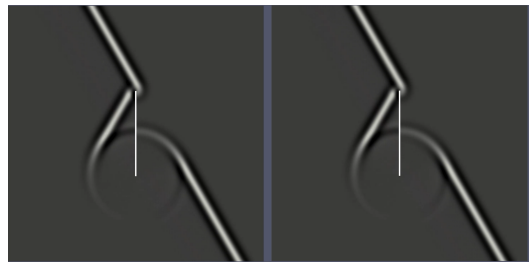

$\theta_{b}=120^{\circ}, t=2.0 \mathrm{~s}$

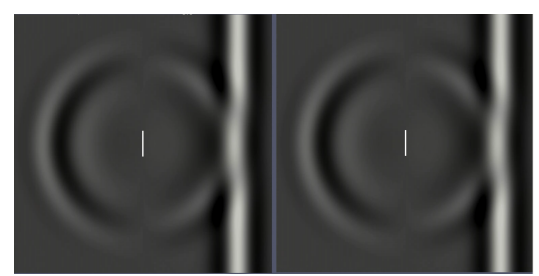

$\theta_{b}=90^{\circ}, t=10.29 \mathrm{~s}$

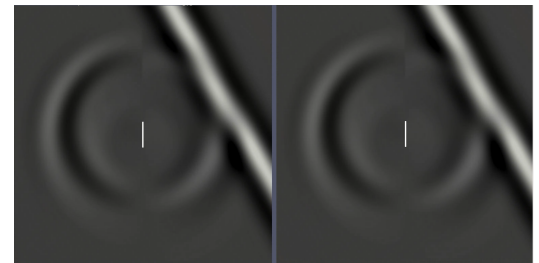

$\theta_{b}=120^{\circ}, t=10.29 \mathrm{~s}$

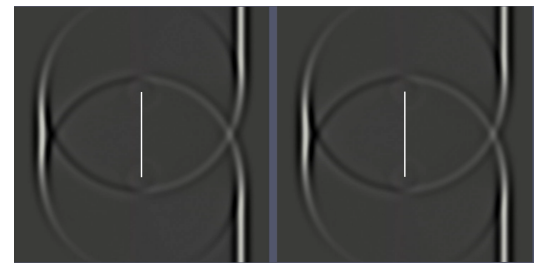

$\theta_{b}=90^{\circ}, t=2.875 \mathrm{~s}$

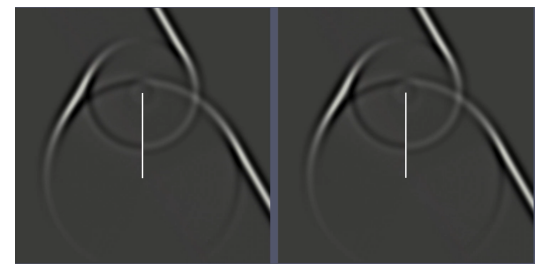

$\theta_{b}=120^{\circ}, t=2.6 \mathrm{~s}$

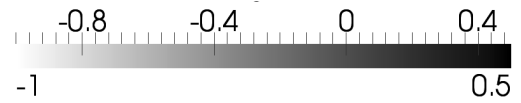

Figure 5: Snapshots of the propagation patterns for a single crack under an incident plane wave in the form of a Ricker pulse obtained with the current SBD method (left) and the BEM algorithm (right). The first two rows correspond to a characteristic dimensionless frequency of the pulse $\eta_{c}=0.5$ for incident angles of $\theta_{b}=90^{\circ}$ and $\theta_{b}=120^{\circ}$ while the last two rows correspond to a characteristic dimensionless frequency of the pulse $\eta_{c}=5.0$ and the incidence angles of $\theta_{b}=90^{\circ}$ and $\theta_{b}=120^{\circ}$. 


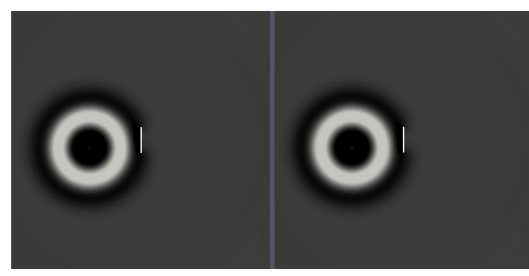

$t=2.94 s$

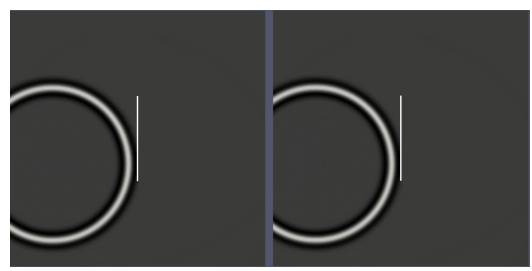

$t=1.1 \mathrm{~s}$

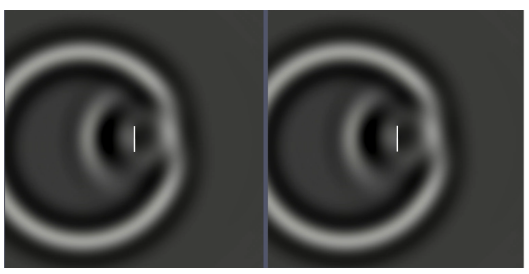

$t=5.39 \mathrm{~s}$

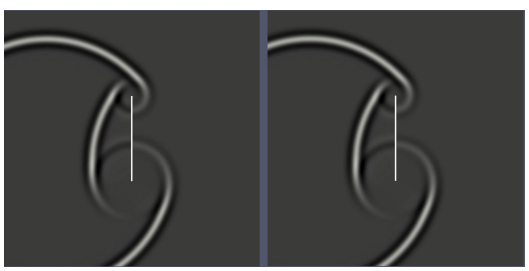

$t=1.67 \mathrm{~s}$

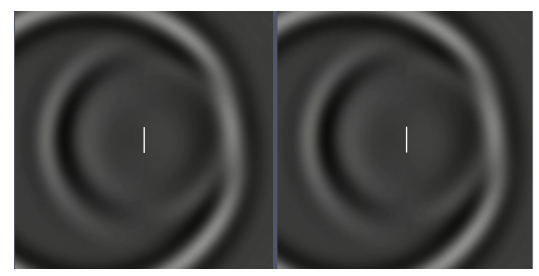

$t=7.56 s$

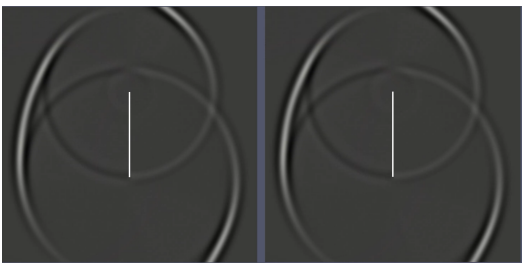

$t=2.5 \mathrm{~s}$

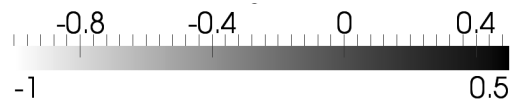

Figure 6: Snapshots of the propagation patterns for a single crack under an incident cylindrical wave in the form of a Ricker pulse obtained with the current SBD method (left) and the BEM algorithm (right). At the low frequency regime the pulse is characterized by its central frequency $\eta_{c}=0.5$, a total time window of $t_{t o t}=13.0 \mathrm{~s}$ and initial time $t_{i n i}=2.0 \mathrm{~s}$. The sources were located at $\left(x_{s}, y_{s}\right)=(-2.0,0.3)$ (first row). At the high frequency regime the pulse had a central frequency $\eta_{c}=5.0$ a total time window $t_{t o t}=4.2 \mathrm{~s}$ and initial time $t_{\text {ini }}=0.2 \mathrm{~s}$ with source located at $\left(x_{s}, y_{s}\right)=(-1.0,0.3)$ (second row).

Figure 7 shows synthetic seismograms for receivers located in the shadowed surface of the crack computed with the SBD and the BEM algorithm for different orders of diffraction. The results from the SBD technique and the numerical solution have been superimposed. It is observed that the SBD method predicts results in good agreement with the numerical solution for even when the considered diffraction is of second order. In fact, the small differences between the solutions derived with both methods are due to the small artificial thickness that must be introduced in the BEM mesh. In the SBD technique the crack is represented like a zero-thickness internal surface. The same response is observed from the synthetic seismograms and time histories displayed in fig. 8. 


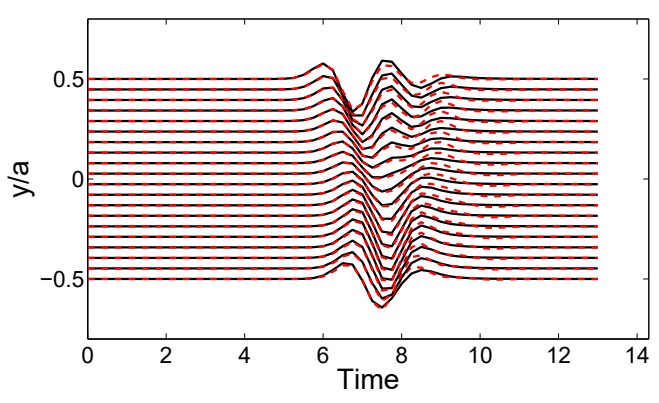

$\theta_{b}=120^{\circ} .1$ order of difraction
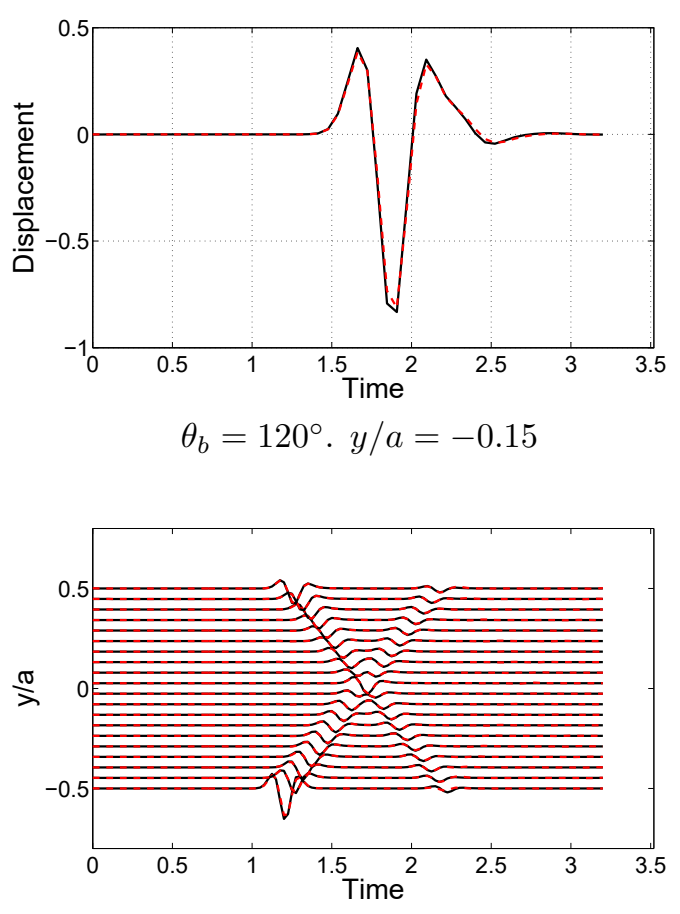

$\theta_{b}=90^{\circ} .1$ order of difraction

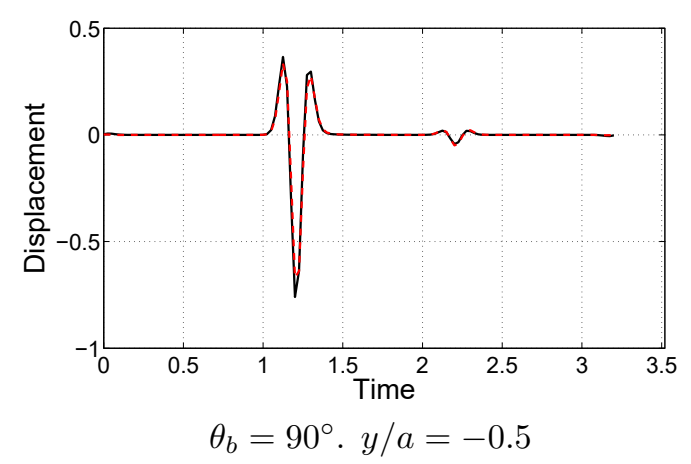

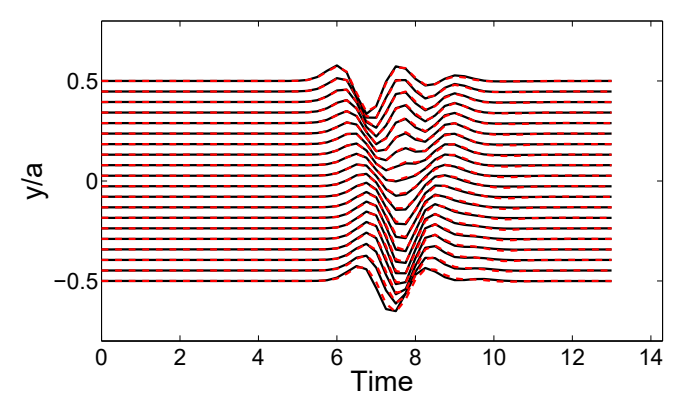

$\theta_{b}=120^{\circ} .2$ orders of difraction
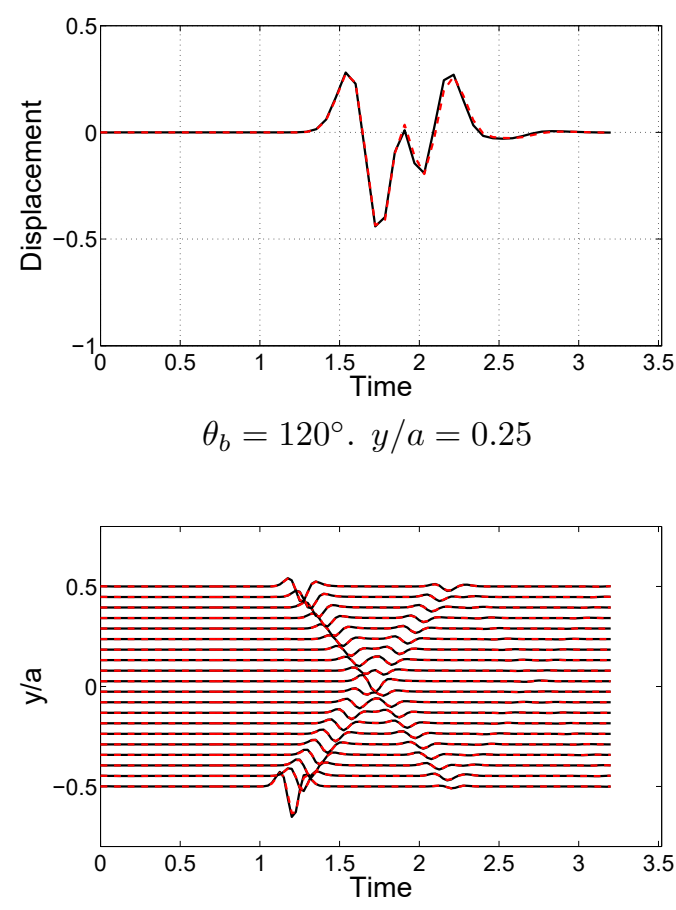

$\theta_{b}=90^{\circ} .2$ orders of difraction

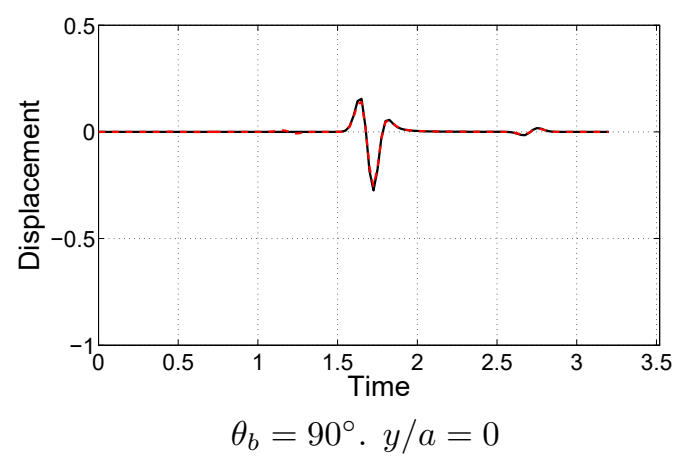

Figure 7: Synthetic seismograms for receivers over the shadowed crack surface for an incident plane wave in the form of a Ricker pulse with high and low central frequencies obtained with the SBD technique (dashed lines) and the BEM algorithm (continuous line). The results in rows 1 and 2 are for a pulse defined by $\eta_{c}=0.5, t_{t o t}=13.0 \mathrm{~s}, t_{i n i}=7.0 \mathrm{~s}$ and those in rows 3 and 4 are for a pulse defined by $\eta_{c}=5.0, t_{t o t}=4.2 s, t_{i n i}=1.7 \mathrm{~s}$. The results were obtained with first and second order diffraction terms. 


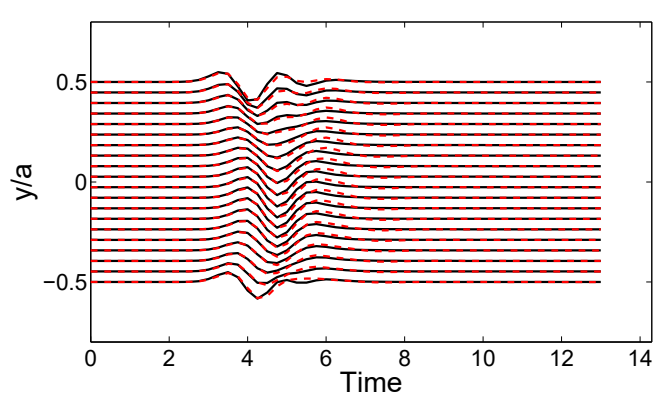

1 order of difraction
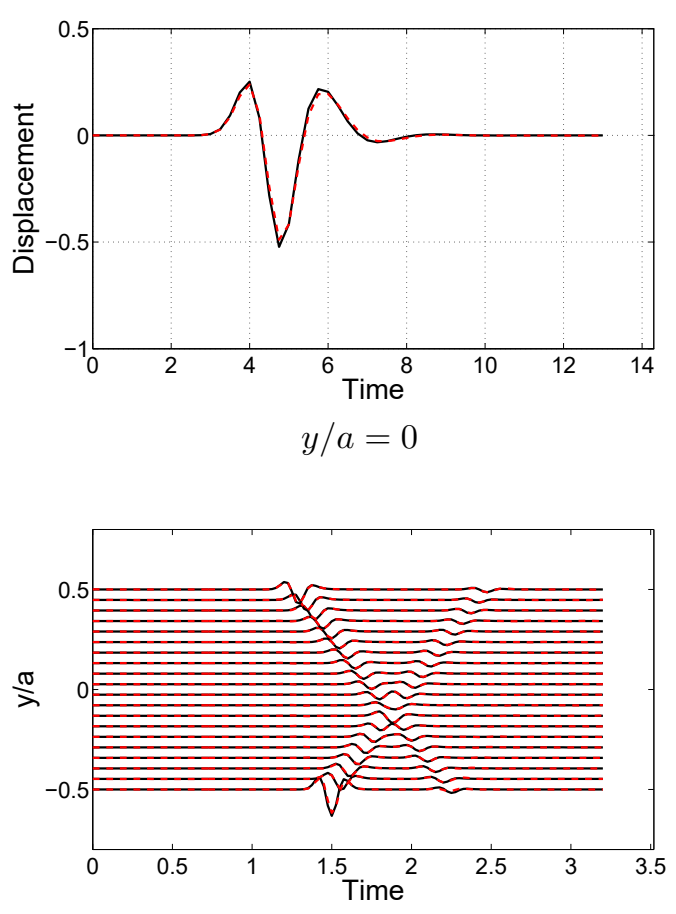

1 order of difraction

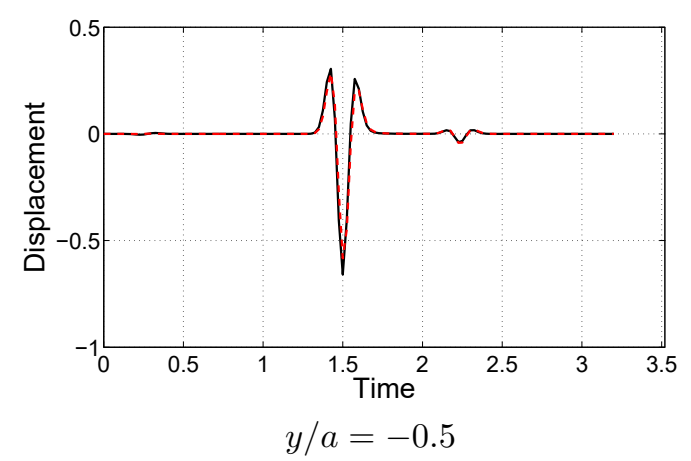

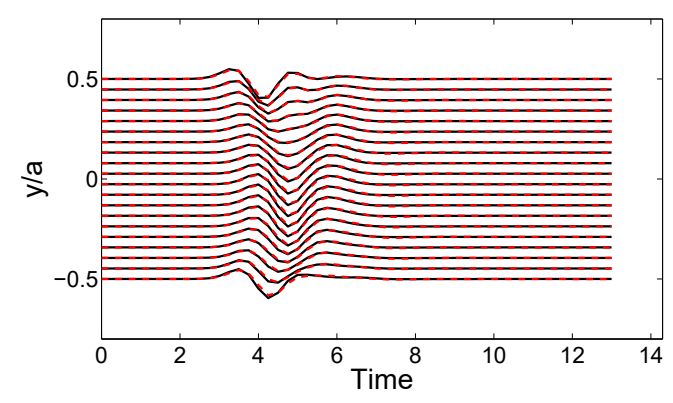

2 orders of difraction
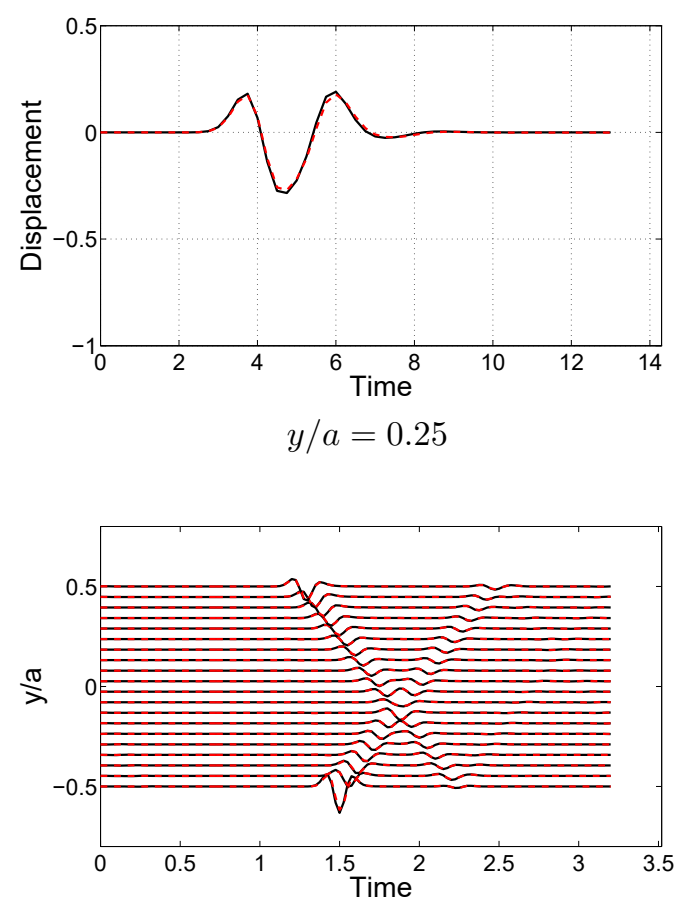

2 orders of difraction

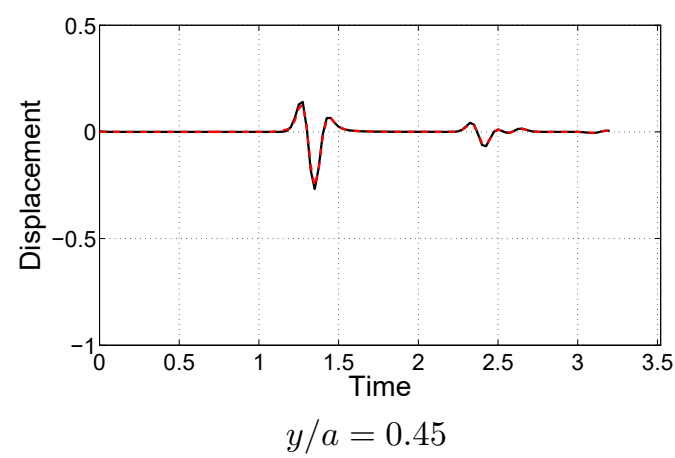

Figure 8: Synthetic seismograms for receivers over the shadowed crack surface for a incident cylindrical wave in the form of a Ricker pulse with high and low central frequencies obtained with the SBD technique (dashed lines) and the BEM algorithm (continuous line). The results in rows 1 and 2 are for a pulse defined by $\eta_{c}=0.5, t_{t o t}=13.0 s, t_{i n i}=7.0 s$ and those in rows 3 and 4 are for a pulse defined by $\eta_{c}=5.0, t_{t o t}=4.2 s, t_{i n i}=1.7 \mathrm{~s}$. The results were obtained with first and second order diffraction terms and the source was located at $\left(x_{s}, y_{s}\right)=(-1.0,0.3)$. The results were obtained with first and second order diffraction terms. 
As an additional validation we compare in fig. 9 our results with those reported by SánchezSesma \& Iturrarán-Viveros (2001). These authors showed how their solution method, which is based on the solution by Sommerfeld (1896), loses accuracy in the low frequency regime due to the plane wave approximation used for the higher order diffracted waves. In order to test the level of error introduced by that plane wave assumption, these authors compared their results with those obtained from the recursive application of the solution for a generalized wedge formulated in MacDonald (1902). Here we compare our results with those from SánchezSesma \& Iturrarán-Viveros (2001). In the plot we used the same notation as in Sánchez-Sesma \& Iturrarán-Viveros (2001) where $r$ represents the radial coordinate of any point along the illuminated zone of the crack and $r_{0}$ is the location of a source point in the shadow zone of the crack, i.e., $r_{0}=a$. In the plot we describe the behaviour of the amplitude of the transfer function for a diffracted wave travelling along the illuminated zone produced by a plane or cylindrical wave travelling along the shadow zone. The comparison corresponds to 4 different frequencies, namely $\kappa r_{o}=\pi / 2, \pi, 2 \pi, 3 \pi$. The good agreement between the results from the SBD technique and the solution from Sánchez-Sesma \& Iturrarán-Viveros (2001), at high and low frequency values is clearly observed. Note that at low frequencies, our SBD solution diffracted waves assumed like plane fronts coincides with the results from Sánchez-Sesma \& Iturrarán-Viveros (2001), but departs from our solution with diffracted cylindrical waves.

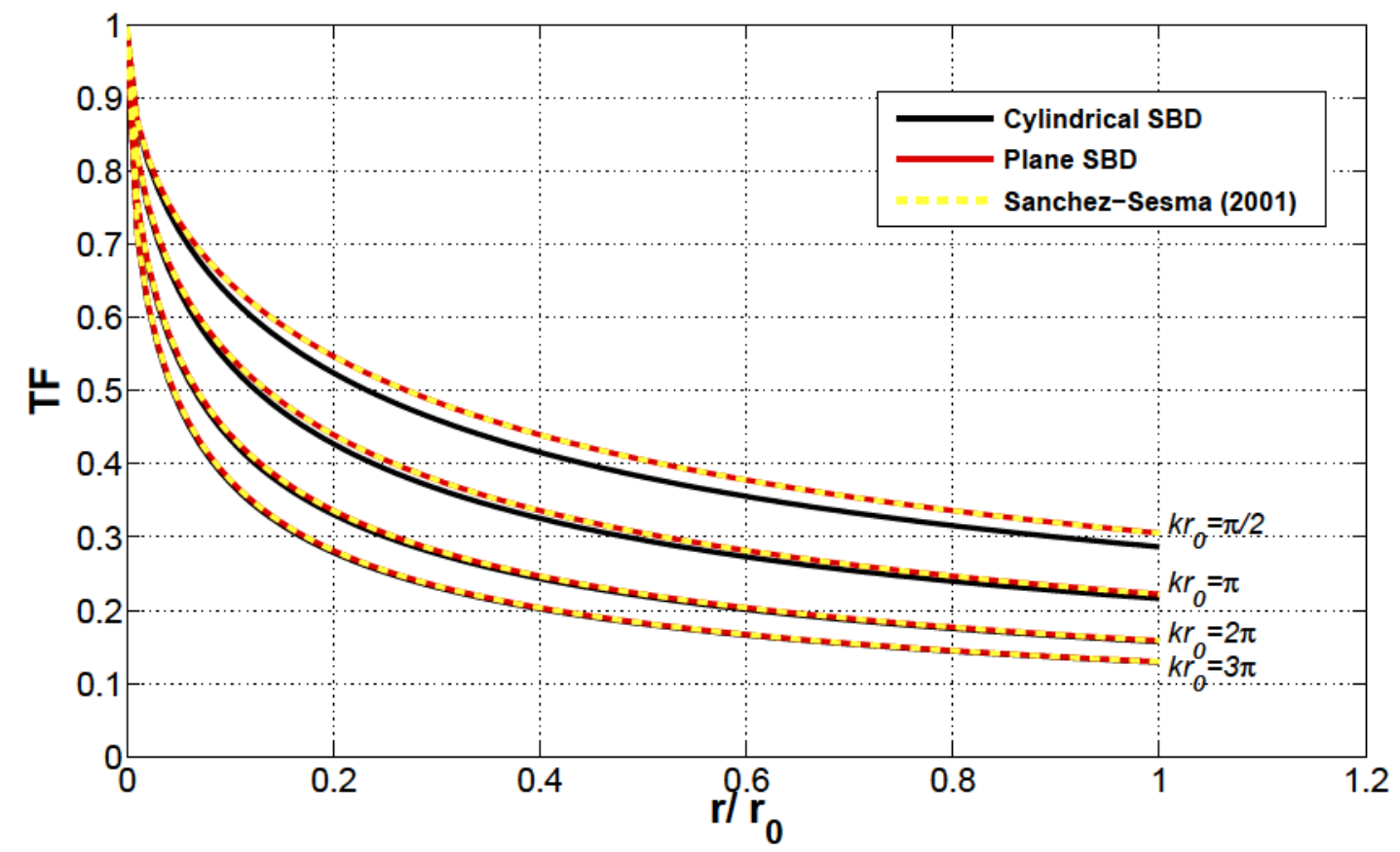

Figure 9: Comparison between the results from the SBD technique and those obtained with the solution from MacDonald (1902) using cylindrical diffracted waves.

\section{Conclusions}

A series solution to the scattering of plane and cylindrical horizontally polarized $S H$ shear waves incident against a crack of finite length embedded in a full-space was constructed. The 
solution was obtained using the superposition of incident, reflected and diffracted rays. This last term in the total field was calculated using the canonical solution for the diffraction of electromagnetic waves by the tip of an infinite wedge due to Kouyoumjian \& Pathak (1974) and its generalized form by Jaramillo et al. (2013). The complete diffracted field for the finite crack, was built through the superposition of two opposite infinite wedges. The solution is shown to be general enough as it is valid for plane waves incident from arbitrary angles and for cylindrical waves with sources at arbitrary locations. On the other hand, in the proposed solution, the waves diffracted by the tips of the crack are propagated against the opposite tip as cylindrical fronts yielding an accurate solution at the low and high frequency regime. In contrast to alternative solutions represented as series of infinite terms, where accuracy is treated in terms of the convergence of the series, in our method accuracy is related to physically based diffraction sources in terms of higher order diffraction contributions. As such it is controlled on a physical basis with the number of required terms shown to depend on the frequency content of the incident wave. At high frequencies only a small number of terms is needed since the amplitude of the diffracted waves dies off with distance reaching a vanishing value as it interacts with the opposite corner. Also, since in the shown solution the incident and reflected rays are represented in closed-form, as opposite to solutions based on separation of variables where the whole field is represented by the infinite series, the solution can be constructed to a desired accuracy with only the consideration of a few terms. Moreover, we propose an empirical rule to obtained the number of terms required to obtain a solution within a specific tolerance depending on the dimensionless frequency. On the other hand, for the particular case of normal incidence, the solution is reduced to a single expression which is found after taking advantage of the symmetry of the problem. Due to the low computational cost involved in the evaluation of the different terms from the series and to their physical nature, this solution is very appealing for use in the validation of numerical implementations and to study propagation of waves in fractured media. Finally, a comparison between the results for plane wave incidence and those for a cylindrical wave reveal that the diffracted field in the shadow zone is independent of the type of incident wave.

\section{References}

Abo-Zena, A. \& King, C., 1973. Sh pulse in an elastic wedge, Bulletin of the Seismological Society of America, 63(5), 1571-1582.

Achenbach, J. D., Gautesen, A. K., \& McMaken, H., 1982. Ray methods for waves in elastic solids: with applications to scattering by cracks, Pitman Advanced Pub. Program.

Caleap, M., Aristégui, C., \& Angel, Y. C., 2007. Further results for antiplane scattering by a thin strip, The Journal of the Acoustical Society of America, 122, 1876.

Chen, B. J., Du, C. L., Zhang, J. L., \& Xiao, Z. M., 2013. Scattering of sh waves by an arbitrarily orientated closed crack, Acta Mech, 224, 2649-2662.

Chen, T., Fehler, M., Fang, X., Shang, X., \& Burns, D., 2012. Sh wave scattering from 2-d fractures using boundary element method with linear slip boundary condition, Geophysical Journal International, 188(1), 371-380.

De Hoop, A. T., 2000. Transient two-dimensional kirchhoff diffraction of a plane elastic sh wave by a generalized linear-slip fracture*, Geophysical Journal International, 143(2), 319-327. 
Garnier, C., Pastor, M.-L., Eyma, F., \& Lorrain, B., 2011. The detection of aeronautical defects in situ on composite structures using non destructive testing, Composite structures, 93(5), 1328-1336.

Hudson, J., 1963. Sh waves in a wedge-shaped medium., The Geophysical Journal of the Royal Astronomical Society, 7(5), 517-546.

Iturrarán-Viveros, U., Vai, R., \& Sánchez-Sesma, F. J., 2005. Scattering of elastic waves by a 2-d crack using the indirect boundary element method (ibem), Geophysical Journal International, 162(3), 927-934.

Iturrarán-Viveros, U., Vai, R., \& Sánchez-Sesma, F. J., 2010. Diffraction of sh cylindrical waves by a finite crack: an analytical solution, Geophysical Journal International, 181(3), $1634-1642$.

Jaramillo, J., Gomez, J., Saenz, M., \& Vergara, J., 2013. Analytic approximation to the scattering of antiplane shear waves by free surfaces of arbitrary shape via superposition of incident, reflected and diffracted rays, Geophysical Journal International, 192(3), 11321143.

Keller, J., 1956. Diffraction by a convex cylinder, IEEE Transactions on Antennas and Propagation., AP-24, AP-24, 312-321.

Keller, J., 1957. Diffraction by an aperture, Journal of Applied Physics, 28(4), 426-444.

Keller, J., 1962. Geometrical theory of diffraction, Journal of the Optical Society America, 52(2), 116-130.

Kouyoumjian, R. \& Pathak, P., 1974. A uniform geometrical theory of diffraction for an edge in a perfectly conducting surface., Proceedings of the IEEE, 62(11), 1448-1461.

Krüger, O. S., Saenger, E. H., \& Shapiro, S. A., 2005. Scattering and diffraction by a single crack: an accuracy analysis of the rotated staggered grid, Geophysical Journal International, 162(1), 25-31.

MacDonald, H., 1902. Electric Waves, Cambridge University Press.

Mow, C.-C. \& Pao, Y.-H., 1971. The diffraction of elastic waves and dynamic stress concentrations, Rand Corporation.

Murai, Y., 2007. Scattering attenuation, dispersion and reflection of sh waves in twodimensional elastic media with densely distributed cracks, Geophysical Journal International, 168(1), 211-223.

Pérez-Ruiz, J., Luzón, F., \& García-Jerez, A., 2007. Scattering of elastic waves in cracked media using a finite difference method, Studia Geophysica et Geodaetica, 51(1), 59-88.

Sanchez-Sesma, F., 1990. Elementary solutions for response of a wedge-shaped medium to incident sh and sv waves., Bulletin of the Seismological Society of America, 80(3), 737-742.

Sánchez-Sesma, F. J. \& Iturrarán-Viveros, U., 2001. Scattering and diffraction of sh waves by a finite crack: an analytical solution, Geophysical Journal International, 145(3), 749-758.

Sommerfeld, A., 1896. Mathematische theorie der diffraktion., Math.Ann, 47, 317-374. 
Tsaur, D., 2010. Exact scattering and diffraction of antiplane shear waves by a vertical edge crack., Geophysical Journal International, 181, 1655-1664.

Waterman, P. C. \& Truell, R., 1961. Multiple scattering of waves, Journal of Mathematical Physics, 2, 512 . 